\title{
Titrated mandibular advancement versus positive airway pressure for sleep apnoea
}

\author{
F. Gagnadoux*, B. Fleury", B. Vielle ", B. Pételle ${ }^{+}$, N. Meslier*, X.L. N'Guyen\#, \\ W. Trzepizur* and J.L. Racineux*
}

ABSTRACT: The aim of this study was to compare mandibular advancement device (MAd) therapy and continuous positive airway pressure (CPAP) for obstructive sleep apnoea/hypopnoea syndrome (OSAHS) after one-night polysomnographic (PSG) titration of both treatments.

59 OSAHS patients (apnoea/hypopnoea index (AHI): $34 \pm 13$ events $\cdot h^{-1}$; Epworth scale: $10.6 \pm 4.5)$ were included in a crossover trial of 8 weeks of MAd and 8 weeks of CPAP after effective titration. Outcome measurements included home sleep study, sleepiness, health-related quality of life (HRQoL), cognitive tests, side-effects, compliance and preference.

The median (interquartile range) AHI was $2(1-8)$ events $\cdot h^{-1}$ with CPAP and 6 (3-14) events $\cdot h^{-1}$ with MAd $(p<0.001)$. Positive and negative predictive values of MAd titration PSG for treatment success were $85 \%$ and $45 \%$, respectively. Both treatments significantly improved subjective and objective sleepiness, cognitive tests and HRQoL. The reported compliance was higher for MAd $(p<0.001)$ with $>70 \%$ of patients preferring this treatment.

These results support titrated MAd as an effective therapy in moderately sleepy and overweight OSAHS patients. Although less effective than CPAP, successfully titrated MAd was very effective at reducing the $\mathrm{AHI}$ and was associated with a higher reported compliance. Both treatments improved functional outcomes to a similar degree. One-night titration of MAd had a low negative predictive value for treatment success.

KEYWORDS: Continuous positive airway pressure, mandibular advancement, obstructive sleep apnoea, titration, treatment

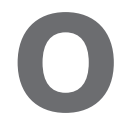

bstructive sleep apnoea/hypopnoea syndrome (OSAHS) is a highly prevalent disease [1] characterised by recurrent episodes of partial or complete obstruction of the upper airways during sleep. Nasal continuous positive airway pressure (CPAP) is the primary treatment of OSAHS, but many patients are unable or unwilling to comply with this treatment. Of OSAHS patients in whom CPAP is recommended, 5-50\% reject this treatment and $12-25 \%$ of the remaining patients can be expected to discontinue CPAP, especially if they have mild OSAHS and/or if they are not "subjectively sleepy" [2, 3]. Mandibular advancement device (MAd) therapy has emerged over the last decade as an alternative therapy for OSAHS [4]. Randomised control trials have demonstrated a reduction of the apnoea/hypopnoea index (AHI) and an improvement of daytime sleepiness with MAd therapy $[5,6]$. In most randomised studies evaluating MAd therapy in OSAHS, the degree of mandibular advancement (MA) was arbitrarily set without any titration procedure, for example at $80 \%$ of the maximal comfortable MA $[5,7]$.
A dose-dependent effect of MA on the AHI, nocturnal oxygen desaturations and pharyngeal collapsibility has been previously demonstrated [8-10], suggesting the potential benefit of an individual MA titration in patients with OSAHS. Comparative studies of MAd and CPAP should therefore include a titration procedure for both treatments. In a pilot study [11], it was demonstrated that it is possible to mobilise the mandible during polysomnography without waking the patient during the advancement manoeuvres. The simple propulsion system that was used in this study allowed one-night titration of the effective MA away from the patient's bedside and prediction of the capacity of MAd to reduce AHI. To the best of our knowledge, no published randomised study has evaluated MAd therapy in OSAHS after polysomnographic (PSG) titration of the effective MA.

The aim of this multi-site, randomised crossover study was to compare 8 weeks of MAd therapy and 8 weeks of CPAP in a mixed-severity group of patients with OSAHS in terms of efficacy, reported side-effects, compliance and preference,
AFFILIATIONS

*Département de Pneumologie, CHU, and

"Unité de Statistiques,

BioMathématiques et Informatique, CHU, Angers,

\# Service de Pneumologie and +Service d'ORL, Hôpital SaintAntoine, Paris, France.

CORRESPONDENCE

F. Gagnadoux

Département de Pneumologie $\mathrm{CHU}$

Angers

France

E-mail: frgagnadoux@chu-angers.fr

Received:

Sept 292008

Accepted after revision:

Feb 182009

First published online:

March 262009 
after one-night PSG titration of both effective MA and CPAP pressure.

\section{METHODS}

\section{Patients and study protocol}

Patients aged 18-70 yrs with OSAHS newly diagnosed by PSG were recruited from the departments of Pulmonary Medicine of Angers University Hospital (Angers, France) and SaintAntoine Hospital (Paris, France). Inclusion criteria were an AHI between 10 and 60 events $\cdot h^{-1}$ and two or more symptoms of OSAHS, including snoring, witnessed apnoea or complaint of daytime sleepiness. Exclusion criteria were previous treatment for OSAHS, body mass index $(\mathrm{BMI}) \geqslant 35 \mathrm{~kg} \cdot \mathrm{m}^{-2}$, a coexisting sleep disorder other than OSAHS, inadequate dental structure or temporomandibular joint disease contraindicating MAd treatment as assessed by a dentist, unstable medical illness and severe sleepiness which may constitute risk to self or others. At baseline, patients underwent anthropometric measurements and individual custom fitting of MAd. Each patient then underwent two consecutive in-laboratory PSGs separated by 1 week in a randomised order for CPAP and MA titration. PSG titration was considered to be ineffective in the case of intolerance of CPAP or MAd and/or inability to achieve $a \geqslant 20 \%$ reduction in $\mathrm{AHI}$. This relatively low threshold for AHI reduction during MA titration was based on our pilot study [11], demonstrating a further reduction in AHI between the titration PSG and the treatment PSG. Patients with effective titration for both CPAP and MAd were randomised for treatment order and were asked to use each treatment for 2 months. The two treatment periods were separated by a 1-week washout period. Outcomes were to be measured during the last week of each treatment period. Outcome measurements included home-limited sleep study, sleepiness, health-related quality of life (HRQoL), cognitive tests, reported side-effects, treatment compliance, satisfaction and preference.

The study was approved by the University of Angers ethics committee and patients gave their informed consent.

\section{CPAP and MAd treatments}

CPAP titration was conducted during PSG according to our standard procedure [12], and patients were then treated at the manually titrated pressure with a CPAP device (Sullivan S6 Elite $^{\mathrm{TM}}$; Resmed, Bella Vista, NSW, Australia) equipped with a microprocessor and pressure monitor, providing a precise index of daily use by measuring the time spent with the mask on.

For MAd therapy, we used a previously described adjustable bi-bloc acrylic oral appliance (AMC ${ }^{\mathrm{TM}}$; Artech Médical, Pantin, France) (fig. 1) [10] with attachments of various sizes allowing MA adjustment. The maximum MA while awake was determined for each patient on three consecutive voluntary manoeuvres. MA titration was performed during PSG after 1 week of acclimatisation to MAd at $50 \%$ of the maximum MA. In the pilot study [11], the arches of the MAd were connected by two lateral hydraulic systems. In order to decrease the dimensions of the appliance in the mouth and to improve comfort during titration, the hydraulic system was replaced by two thin plastic-coated metal cables connected to the upper and lower dental arches. A modified infusion pump (Artech Médical) was used to perform remote progressive MA via a computer interface program (Cidelec, Angers, France). Using this propulsion system, MA was increased by 1-mm increments every $15 \mathrm{~min}$ until a significant reduction of the incidence of the sleep-disordered breathing was obtained or until reaching the maximum advancement position was reached (i.e. $150 \%$ of maximum MA) or the position caused discomfort or pain, waking the patient and preventing any further progression. The length of the MAd attachments was then adjusted to obtain the optimal advancement reached during the titration night.

\section{Sleep recordings}

In-laboratory PSG (CID $102^{\mathrm{TM}}$; Cidelec) was performed as previously described [12] and scored according to standard criteria [13] using nasal pressure cannulae and tracheal sounds (suprasternal microphone) for airflow measurement. Homelimited sleep studies (CID $102 \mathrm{~L}^{\mathrm{TM}}$; Cidelec) under CPAP and MAd included nasal pressure cannulae, tracheal sounds and arterial oxygen saturation $\left(\mathrm{Sa}_{2} \mathrm{O}_{2}\right)$ (finger pulse oximetry). Respiratory events were scored manually. Apnoea was defined as cessation of airflow for $\geqslant 10 \mathrm{~s}$. Hypopnoea was defined as a $>50 \%$ reduction of airflow or a $<50 \%$ reduction of airflow accompanied by a $3 \%$ decrease in $\mathrm{Sa}_{1} \mathrm{O}_{2}$.

\section{Measures of sleepiness, cognitive function and HRQOL}

Subjective sleepiness was assessed by the Epworth sleepiness scale (ESS) [14]. Objective sleepiness was measured using the Oxford sleep resistance (OSLER) test with calculation of the sleep latency and the number of errors [15]. Attention and concentration were also investigated using the Trail Making A (TMT A) and B (TMT B) cognitive tests [16]. HRQoL was evaluated using the Nottingham Health Profile (NHP) [12]. Each subject completed a single OSLER test at 09:00 h after a quiet period of filling in questionnaires with the investigator. Measures of sleepiness, HRQoL and cognition were performed at baseline for MAd and CPAP.

\section{Treatment related side-effects, compliance, satisfaction and preference}

At the end of each treatment period, patients were asked to give a score from 0 to 3 (0, absent; 1 , mild; 2, moderate;

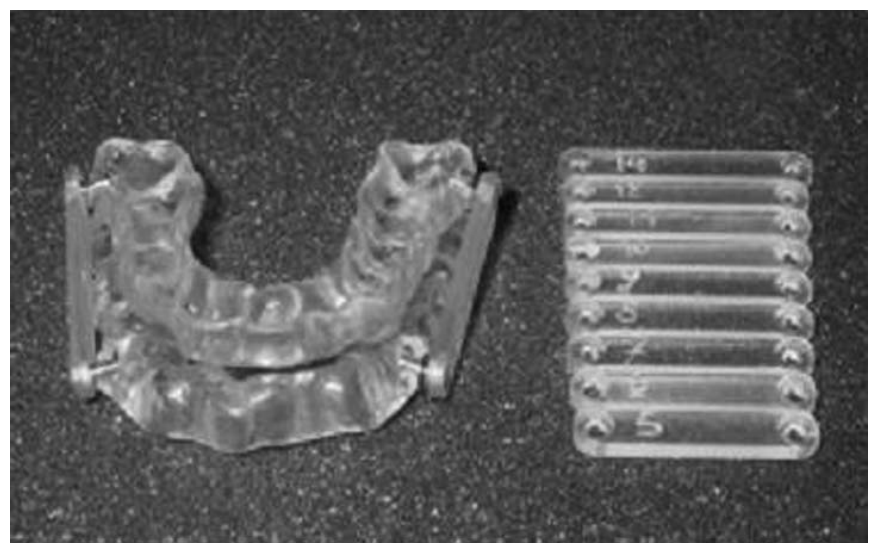

FIGURE 1. The mandibular advancement device (AMC ${ }^{\mathrm{TM}}$; Artech Médical, Pantin, France) used in the study. Full-coverage acrylic appliances designed to fit to the upper and lower dental arches (left) are connected by acrylic plates of increasing length (right) 
3, severe) regarding six common side-effects of CPAP (congested, drippy or irritated nose, skin lesion, irritated eyes and dry mouth) and MAd (jaw pain, tooth pain, muscle stiffness, dry mouth, hypersalivation and occlusal change). A mean side-effects score from 0 to 18 was then calculated for each treatment. Compliance with CPAP and MAd was assessed by self-reporting. Objective data regarding CPAP compliance were downloaded from the internal memory of the device. Global treatment satisfaction was assessed using a $0-10$ visual analogue scale. At the end of the study, patients were asked to indicate their preferred treatment.

\section{Statistical analysis}

Continuous variables were described as mean \pm SD for variables with a normal distribution and median (interquartile range) for variables with a non-normal distribution. Normality of distribution was assessed using the Kolmogorov-Smirnov test. A sample size of 60 patients was calculated to detect a $1 \mathrm{SD}$ difference in AHI between the two treatments with a power of $99 \%$ and a significance level of 5\% (two-sided). Betweentreatment differences were assessed using a paired t-test and unpaired t-test for variables with a normal distribution, and by a Wilcoxon signed-rank test and Mann-Whitney test for variables with a non-normal distribution. Treatment-by-period interaction (carry-over effect) was tested by ANOVA for repeated measures. The correlation between continuous variables was assessed by Spearman's rank correlation coefficient. All reported p-values are two-sided and the Bonferroni correction was used for pairwise comparisons. A p-value of $\leqslant 0.05$ was considered to indicate statistical significance. All analyses were performed using SPSS version 15.0 statistical software (SPSS, Chicago, IL, USA).

\section{RESULTS}

A flow diagram summarising the distribution of the subjects is shown in figure 2. 69 patients underwent PSG titration of both MAd and CPAP. Comparison of sleep data during CPAP and MAd titration showed no significant difference in sleep efficiency $(83.7 \pm 8.2 \%$ versus $82.7 \pm 12 \%)$, stage $1-2$ sleep ( $58.3 \pm 12.1 \%$ versus $56.8 \pm 13.7 \%$ of total sleep time), stage $3-4$ sleep $(21.5 \pm 9.1 \%$ versus $21 \pm 9.1 \%$ of total sleep time), rapid eye movement (REM) sleep $(20.2 \pm 6.7 \%$ versus $22.2 \pm 7.8 \%$ of total sleep time) and micro-arousal index (15.9 \pm 10.5 versus $15.5 \pm 10$ events $\cdot h^{-1}$ of sleep). MA titration was ineffective in eight patients. Five patients were intolerant to progressive MA with jaw pain, discomfort and/or repeated awakenings preventing further progression. In three patients, it was impossible to achieve $\mathrm{a} \geqslant 20 \%$ reduction in $\mathrm{AHI}$ at the position of maximum MA. Two patients did not tolerate CPAP during the titration PSG. A total of 59 patients with successful titration of both CPAP (mean effective pressure: $9.1 \pm 1.7 \mathrm{mmH}_{2} \mathrm{O}$ ) and MAd (mean effective MA: $9.8 \pm 2.1 \mathrm{~mm}$ corresponding to $103 \pm 20 \%$ of the maximum voluntary advancement) were randomised for treatment sequence. Patients' characteristics at the time of enrolment are shown in table 1. 25 patients had mild-to-moderate OSAHS with AHI between 11 and 29 events $\cdot \mathrm{h}^{-1}$, and 34 patients had severe OSAHS with AHI between 30 and 60 events $\cdot h^{-1}$. Of these, 30 were randomised to MAd for 2 months followed by a 1-week washout, then CPAP for 2 months. One of these patients withdrew during the MAd period and was lost to follow-up and another withdrew during

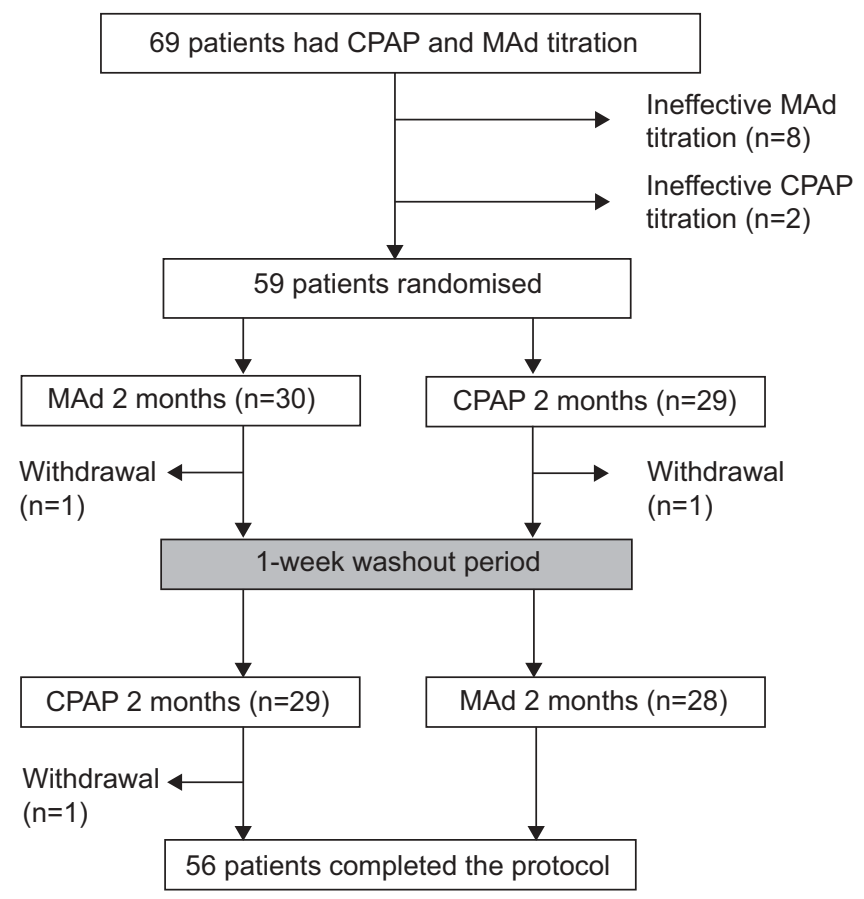

FIGURE 2. Flow diagram of subjects during the study. CPAP: continuous positive airway pressure; MAd: mandibular advancement device.

the CPAP period and refused to undergo any further evaluation. The remaining 28 patients completed the protocol. 29 patients were randomised to the reversed treatment sequence with CPAP for 2 months followed by a 1-week washout, then MAd for 2 months. One of these patients withdrew during the CPAP period and was lost to follow-up; 28 patients completed the protocol. A total of 56 patients completed the study. No significant difference in body weight was observed between values at baseline $(77.8 \pm 11.5 \mathrm{~kg})$, on CPAP $(79.3 \pm 11.4 \mathrm{~kg})$ and on MAd $(78.9 \pm 11.5 \mathrm{~kg})$.

\section{Home-limited sleep studies under CPAP and MAd}

Home sleep studies data under CPAP and MAd in the patients who completed the trial are compared in table 2. No treatmentby-period interaction was observed for any home sleep study variable. CPAP was significantly more effective on the snoring index, AHI and nocturnal oxygenation. Treatment AHI was $<10$ events $\cdot h^{-1}$ for $39(70 \%)$ patients on MAd and 46 events $\cdot h^{-1}$

\begin{tabular}{lcc} 
TABLE 1 & Baseline characteristics of randomised patients \\
Parameter & Mean \pm SD & Range \\
\hline & & \\
Age yrs & $50.3 \pm 9.1$ & $26-69$ \\
Body mass index $\mathbf{~ k g} \cdot \mathbf{m}^{-\mathbf{2}}$ & $26.7 \pm 3.5$ & $20.3-34.6$ \\
Apnoea/hypopnoea index & $34.2 \pm 13.0$ & $11-60$ \\
Epworth sleepiness scale & $10.6 \pm 4.5$ & $0-21$ \\
Maximum mandibular advancement $\mathbf{~ m m}$ & $9.5 \pm 1.5$ & $6-13$ \\
\hline & & \\
\#: $\mathrm{n}=59 ;$ 13 females. & &
\end{tabular}




\begin{tabular}{|c|c|c|c|c|}
\hline \multirow[t]{2}{*}{ TABLE 2} & \multicolumn{4}{|c|}{$\begin{array}{l}\text { Comparison of home sleep study data with } \\
\text { continuous positive airway pressure (CPAP) and } \\
\text { mandibular advancement device (MAd) }\end{array}$} \\
\hline & & CPAP & MAd & $\mathrm{p}$-value \\
\hline \multicolumn{2}{|c|}{ Total recording time $\mathrm{min}$} & $456(384-477)$ & $449(416-476)$ & 0.4 \\
\hline \multicolumn{2}{|c|}{ Snoring index } & $16(2-52)$ & $55(10-149)$ & $<0.001$ \\
\hline \multicolumn{2}{|c|}{ Apnoea/hypopnoea index } & $2(1-8)$ & $6(3-14)$ & 0.001 \\
\hline \multicolumn{5}{|c|}{$3 \%$ oxygen desaturation } \\
\hline \multicolumn{2}{|l|}{ index } & $1.7(0.7-5.1)$ & $6.3(3.0-9.7)$ & $<0.001$ \\
\hline \multicolumn{2}{|c|}{ Mean $\mathrm{Sa}, \mathrm{O}_{2} \%$} & $96(95-96)$ & 94 (93-95) & $<0.001$ \\
\hline
\end{tabular}

$(82 \%)$ patients on CPAP. A complete response $(\geqslant 50 \%$ reduction in $\mathrm{AHI}$ to $<5$ events $\cdot \mathrm{h}^{-1}$ ) was obtained in $73.2 \%$ of patients with CPAP and $42.8 \%$ with MAd. A partial response $\left(\geqslant 50 \%\right.$ reduction in AHI but AHI remaining $\geqslant 5$ events $\left.\cdot \mathrm{h}^{-1}\right)$ was observed in $23.2 \%$ of patients with CPAP and $51.7 \%$ with MAd. Treatment failure $(<50 \%$ reduction in AHI) occurred in $3.5 \%$ of patients with CPAP and $5.3 \%$ with MAd. Figure 3 illustrates the percentages of complete response, partial response and treatment failure on MAd according to baseline OSAHS severity. A complete response on MAd was achieved in $58.3 \%$ of patients with mild-to-moderate OSAHS versus $31.2 \%$ of patients with severe OSAHS. After including the patients who were not randomised due to ineffective titration and those who dropped out of the study, treatment failure occurred in $18.4 \%$ of patients for MAd and $6.6 \%$ for CPAP.

A significant correlation $(\mathrm{r}=0.52, \mathrm{p}<0.001)$ was observed between the AHI determined during MA titration (12 (6-14))

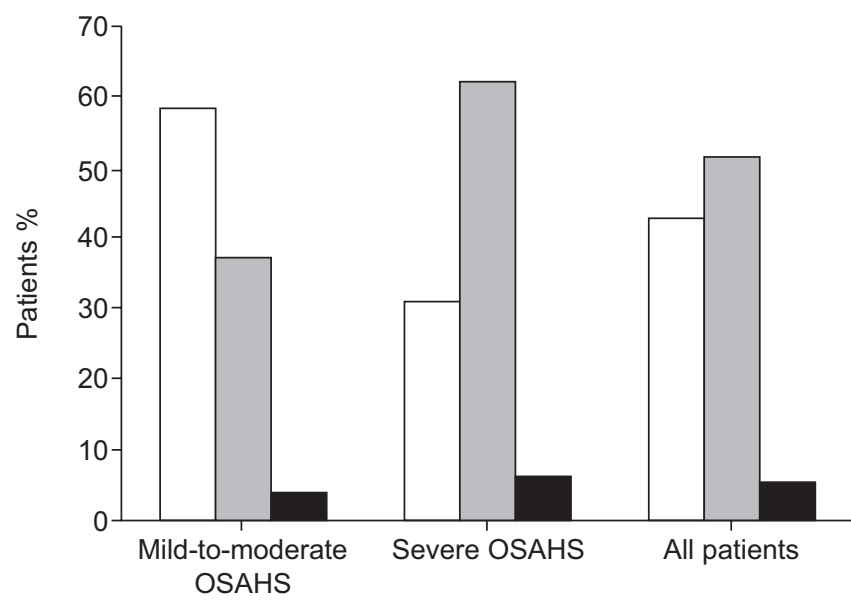

FIGURE 3. The treatment response with mandibular advancement device in all patients who completed the study and according to obstructive sleep apnoea/ hypopnoea syndrome (OSAHS) severity defined as mild-to-moderate (apnoea/ hypopnoea index $(\mathrm{AHI})<30$ events $\left.\cdot \mathrm{h}^{-1}\right)$ and severe $\left(\mathrm{AHI} \geqslant 30\right.$ events $\left.\cdot \mathrm{h}^{-1}\right)$. A complete response $(\square)$ was defined by a $\geqslant 50 \%$ reduction in AHI to $<5$ events $\cdot h^{-1}$, partial response $(\square)$ by a $\geqslant 50 \%$ reduction in the $\mathrm{AHI}$ but with $\mathrm{AHI}$ remaining $\geqslant 5$ events. $\mathrm{h}^{-1}$, and treatment failure ( $\left.\mathbf{\square}\right)$ by $<50 \%$ reduction in $\mathrm{AHI}$. and that obtained during home sleep study with MAd (6 (314)) in the 56 patients who completed the study (fig. 4). Among the 27 patients with $\mathrm{AHI} \leqslant 10$ events $\cdot \mathrm{h}^{-1}$ during MA titration, 23 had an AHI $<10$ events $\cdot h^{-1}$ with MAd on limited sleep study (positive predictive value $85 \%$ ) and four had an AHI $\geqslant 10$ events $\cdot h^{-1}$. Among the 29 patients with AHI $>10$ events $\cdot h^{-1}$ during MA titration, $16(55 \%)$ had an AHI $<10$ events $\cdot \mathrm{h}^{-1}$ on MAd on limited sleep study and 13 had an AHI $\geqslant 10$ events $\cdot h^{-1}$ (negative predictive value $45 \%$ ).

\section{Measures of sleepiness, cognitive function and HRQOL}

In the patients who completed the study, CPAP and MAd both significantly improved subjective and objective daytime sleepiness compared with baseline (table 3). No significant difference was observed between MAd and CPAP for ESS and OSLER test data. CPAP and MAd both significantly improved the TMT A cognitive test with no significant difference between CPAP and MAd values. In contrast, a significant improvement of TMT B was only observed with CPAP. No treatment-by-period interaction was observed for any parameters of sleepiness and cognitive function. HRQoL data assessed by the NHP questionnaire at baseline on MAd and on CPAP are presented in figure 5. For CPAP, a significant improvement was observed for two out of six domains of HRQoL including emotional reaction and energy. For MAd, HRQoL was significantly improved for four out of six domains including physical mobility, pain, emotional reaction and sleep. A significant treatment-by-period interaction was observed for emotional reaction and sleep with a significantly better emotional reaction and subjective sleep quality with MAd for the second treatment period but no difference between treatments for the first treatment period.

\section{Treatment related side-effects, compliance, satisfaction and preference}

The mean side-effects score was similar for MAd and CPAP in the patients who completed the study (table 4). In contrast, reported daily compliance was significantly higher with MAd for both the number of hours of daily use and the percentage of

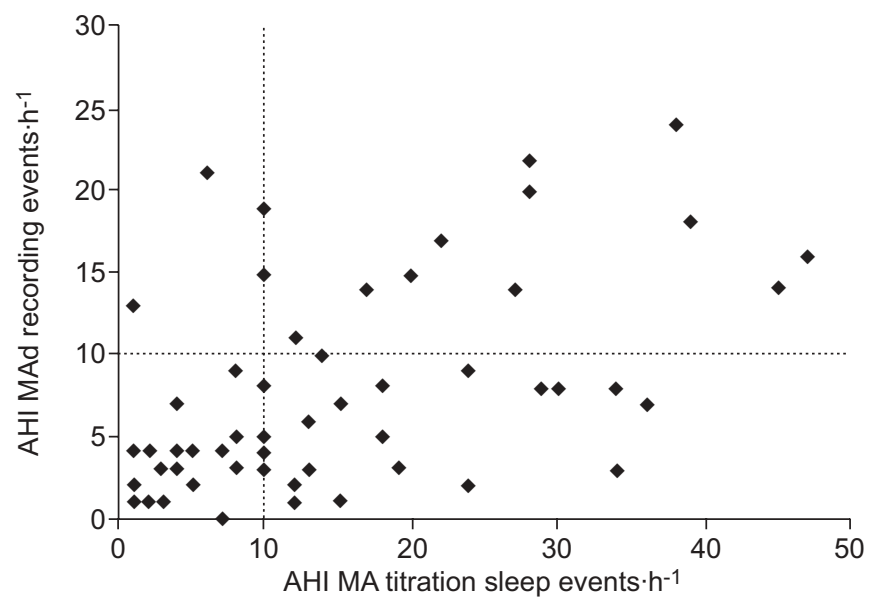

FIGURE 4. Comparison of the apnoea/hypopnoea index (AHI) during mandibular advancement (MA) titration polysomnography and during home-limited sleep study with a mandibular advancement device (MAd). 


\begin{tabular}{|c|c|c|c|c|}
\hline \multirow{2}{*}{$\begin{array}{l}\text { TABLE } 3 \\
\text { Parameter }\end{array}$} & \multicolumn{4}{|c|}{$\begin{array}{l}\text { Measures of sleepiness, and cognitive function } \\
\text { at baseline, on continuous positive airway } \\
\text { pressure (CPAP) and on mandibular } \\
\text { advancement device (MAd) }\end{array}$} \\
\hline & & Baseline & CPAP & MAd \\
\hline \multicolumn{2}{|c|}{ Epworth sleepiness scale $(\downarrow)$} & $10.6 \pm 4.5$ & $8.2 \pm 3.9^{* \star \star}$ & $7.7 \pm 4.0^{* * \star}$ \\
\hline \multicolumn{5}{|l|}{ OSLER test } \\
\hline \multicolumn{2}{|c|}{ Sleep latency s ( $\uparrow)$} & $2094 \pm 674$ & $2300 \pm 391^{*}$ & $2312 \pm 322 *$ \\
\hline \multicolumn{2}{|c|}{ Errors $n(\downarrow)$} & $12.5 \pm 17.9$ & $8.7 \pm 19.8^{\star}$ & $4.3 \pm 7.5^{\text {** }}$ \\
\hline \multicolumn{2}{|c|}{ Trail Making Test $\mathbf{A}(\downarrow)$} & $36.4 \pm 10.9$ & $30.1 \pm 8.9^{\star \star \star}$ & $32.5 \pm 9.6^{*}$ \\
\hline \multicolumn{2}{|c|}{ Trail Making Test B $(\downarrow)$} & $79.3 \pm 26.2$ & $68.5 \pm 22.2^{\star \star *}$ & $73.4 \pm 33.0$ \\
\hline
\end{tabular}

Data are presented as mean $\pm \mathrm{SD}$; ${ }^{\#}$ : the arrows in parentheses indicate the direction of improvement for each parameter. OSLER: Oxford sleep resistance. *: $p<0.05$ for the comparison of treatment versus baseline; ${ }^{* *}: p<0.01$ for the comparison of treatment versus baseline; ${ }^{* \star}$ : $p<0.001$ for the comparison of treatment versus baseline. For each variable, $p$-values are adjusted for multiple comparisons using Bonferroni correction, i.e. p-values are multiplied by 3 .

nights on which treatment was used. No treatment-by-period interaction was observed for reported compliance. For CPAP, comparison of reported compliance with objective data downloaded from the internal memory of the device (6.0 (4.0-7.0) versus $4.0(0.9-5.4) \mathrm{h}$ for daily use and 90 (40-99) versus 79 (4293) for the percentage of nights on treatment, respectively) showed that patients overestimated actual CPAP use. A treatment-by-period interaction was observed for treatment satisfaction with a higher level of satisfaction for MAd during the second treatment period but no difference between the two treatments during the first treatment period. At the end of the

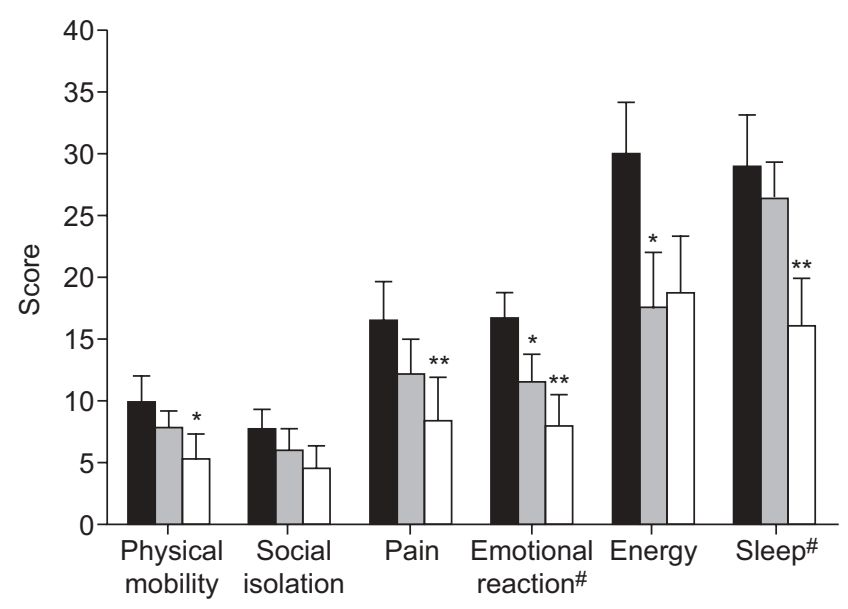

FIGURE 5. Nottingham Health Profile domains of quality of life at baseline ( $\mathbf{\square})$, using continuous positive airway pressure $(\square)$ and using a mandibular advancement device $(\square)$. Data expressed as mean \pm SEM. For each variable, p-values are adjusted for multiple comparisons using Bonferroni correction, i.e. p-values are multiplied by 3 . Lower scores indicate better functioning. ${ }^{*}: p<0.05$ versus baseline; **: $p<0.01$ versus baseline. ${ }^{*}$ : significant treatment-by-period effect. study 42 (71.2\%) out of 55 patients preferred MAd, five $(8.5 \%)$ preferred CPAP and eight had no treatment preference.

\section{DISCUSSION}

The efficacy and acceptance of CPAP and MAd were compared after one-night titration of the two treatments in a mixed-severity group of subjects with newly diagnosed OSAHS. CPAP was more effective than MAd at reducing AHI. Both treatments were associated with a similar improvement in subjective and objective sleepiness, cognitive function tests and HRQoL. Side-effects were similar in frequency and intensity but self-reported compliance was higher with MAd with $>70 \%$ of patients preferring this treatment.

To the best of our knowledge, this is the first randomised study comparing CPAP and MAd after one-night PSG titration of both treatments. Titration of MA was designed to optimise MAd efficacy. Recent reviews on oral appliances for OSAHS treatment concluded that treatment success (AHI $<10$ events $\cdot h^{-1}$ ) was achieved in an average of $52-54 \%$ of treated patients $[6,17]$. By pooling seven randomised control trials comparing CPAP and MAd in 232 OSAHS patients, HOFFSTEIN [17] found a mean AHI of 24 events $\cdot h^{-1}$ at baseline, six on CPAP and 14 on MAd. None of these randomised trials included any MA titration procedure, except for one study using a partly adjustable appliance with progressive titration over the 4-month treatment period [18]. Although CPAP remained superior to MAd in terms of $\mathrm{AHI}$ reduction in the present study, a higher response rate (AHI $<10$ events $\cdot h^{-1}$ in $70 \%$ of patients) and a lower AHI (6 (3-14) events $\left.\cdot h^{-1}\right)$ were observed with MAd than in previous randomised studies [17].

Our one-night MA titration procedure had a high positive predictive value, with $85 \%$ of effective MAd therapy (AHI $<10$ events $\cdot h^{-1}$ ) in patients with AHI $\leqslant 10$ events $\cdot h^{-1}$ during MA titration PSG. In contrast, it had a low (45\%) negative predictive value for treatment success compared with $78 \%$ that was recently obtained by DorT et al. [19]. One possible explanation for this low negative predictive value is a progressive accommodation to MAd resulting in increased efficacy over time. Even in patients with MA titration AHI $>10$ events $\cdot h^{-1}$, a partial response was observed during PSG titration with a $35 \%$ decrease in AHI compared with baseline. Sleep disruption caused by the MAd titration procedure may also have falsely elevated AHI due to a higher percentage of

\begin{tabular}{|c|c|c|c|c|}
\hline TABLE 4 & \multicolumn{4}{|c|}{$\begin{array}{l}\text { Comparison of treatment related side-effects } \\
\text { and compliance on continuous positive airway } \\
\text { pressure (CPAP) and mandibular advancement } \\
\text { device (MAd) }\end{array}$} \\
\hline Parameter & & CPAP & MAd & $p$-value \\
\hline \multicolumn{2}{|c|}{ Mean side-effects score } & $3.2 \pm 3.4$ & $3.2 \pm 3.1$ & 0.8 \\
\hline \multicolumn{5}{|c|}{ Reported compliance } \\
\hline \multicolumn{2}{|c|}{ Hours per night } & $6.0(4.0-7.0)$ & $7.0(6.0-8.0)$ & $<0.001$ \\
\hline \multicolumn{2}{|c|}{ Nights on treatment \% } & 90 (40-99) & $98(90-100)$ & $<0.001$ \\
\hline
\end{tabular}

Data are presented as mean \pm SD or median (interquartile range), unless otherwise stated 
light non-REM sleep or more arousals than during CPAP titration. However, no difference in sleep architecture was observed between CPAP and MAd titration PSG. The denominator for AHI calculation was total sleep time during MA titration and time in bed during home sleep study. This may have erroneously contributed to the decrease in AHI between titration and home sleep study. Finally, it may reflect a technical failure of our propulsion system to predict the longterm therapeutic efficacy of MAd in some patients. The need of a supervised PSG in the sleep laboratory with the presence of a trained technician throughout the night and the low negative predictive value of our titration procedure may potentially reduce its clinical utility in routine clinical practice.

In agreement with previous randomised studies [20-22], no significant difference in terms of ESS improvement was observed between CPAP and MAd. In contrast, two recent trials concluded that there was a lower improvement in ESS under MAd $[7,23]$. The conflict between the current and recent studies $[7,23]$ may be explained by differences in patient characteristics and/or efficacy of MAd to control sleepdisordered breathing. The patients included in the present study were less sleepy (mean ESS 10.6) than those included by ENGLEMAN et al. [7] and LAM et al. [23] (mean ESS 14 and 12, respectively) and MAd was more effective than in the studies by ENGLEMAN et al. [7] and LAM et al. [23], as assessed by the mean AHI on MAd (7.8 versus 15 and 10.6 events $\cdot h^{-1}$, respectively) although baseline AHI was equivalent or even higher (34.2 versus 31 and 21 events $\cdot h^{-1}$, respectively). Two studies evaluated objective sleepiness using the maintenance of wakefulness test (MWT) on CPAP and MAd [7, 22]. In both studies [7, 22], MWT values were not significantly different between the two treatments. Using the OSLER test, we demonstrated a similar improvement in objective daytime alertness with CPAP and MAd. A significant improvement was observed for four of the six domains of HRQoL with MAd and two of the six domains with CPAP with no significant difference between the two treatments. A significant treatmentby-period interaction was observed for two domains of HRQoL, i.e. emotional reaction and sleep with a better emotional reaction and subjective sleep quality with MAd only for the second treatment period. As these variables are assessed subjectively by the patient, it can be hypothesised that the global impression expressed by the patients in the second treatment period was probably modified by comparison with the treatment received during the first treatment period. Finally, the study confirmed previous reports [18, 20, 21, 24] of higher patient preference for MAd. Although reported sideeffects were similar in frequency and intensity, MAd was preferred by $>70 \%$ of patients and was associated with a significantly higher reported compliance.

This study presents a number of potential limitations. The higher rate of ineffective titration with MAd than with CPAP may have biased the results of the comparative study towards a more favourable outcome for MAd than would have otherwise been the case. However, it appeared unacceptable to submit the patients in whom we failed to determine an, at least partially, effective MA during titration PSG to 2 months of MAd treatment. The selective nature of the study population may also have contributed to the discordant results between the present study and previous randomised trials [7, 23].
The overall treatment failure rate with MAd including the patients who were not randomised because of ineffective titration or who dropped out of the study was $18 \%$, similar to the failure rate reported in a previous study using the same device, with progressive MA titration based on sequential sleep recordings over several weeks [10]. Our study was a superiority trial and therefore we cannot claim formal equivalence between the two treatments in terms of improvement of functional outcomes. The patients in the present study were moderately sleepy. However, the mean ESS was not different from that reported in a previous study by our group including 263 consecutive OSAHS patients [12]. Although this study was based on a mixed-severity group of subjects with mild-tosevere OSAHS, mean BMI was lower than in previous studies [12]. A higher BMI has been associated with lower efficacy of MAd in several studies [6]. Furthermore, a higher body weight was independently associated with CPAP preference in a previous randomised study [7]. The relatively low BMI of the present population may therefore have contributed to MAd efficacy and preference. The absence of a control period constitutes another potential limitation of this study as a previous randomised controlled trial comprising 3 months of placebo treatment demonstrated a significant improvement after placebo on many of the neurobehavioural tests [22]. A placebo effect may therefore have contributed to improvement of some parameters in our study. As there is no way to record objective daily use of MAd at the present time, CPAP and MAd were compared on the basis of reported daily compliance. As previously described [25], reported use of CPAP significantly overestimated the actual running time of the device. Patients in this study may therefore also have overestimated the actual daily use of MAd. Various dental-skeletal effects have been documented after long-term use of MAd, including changes in the degree of vertical and horizontal overlap of the teeth [17]. No objective assessment of orthodontic change was performed in this study. However, reported side-effects including subjective occlusal changes were reported to be mild and did not constitute an obstacle to long-term regular use of MAd.

In conclusion, despite these potential limitations, the results of our study support titrated MAd as an effective therapy in moderately sleepy and overweight OSAHS patients. Although less effective than CPAP, successfully titrated MAd was very effective at reducing $\mathrm{AHI}$ and was associated with a higher reported compliance than that observed on CPAP. Both treatments improved functional outcomes in a similar way. PSG titration of MA required the presence of a trained technician throughout the night and had a low negative predictive value for treatment success, which may potentially reduce its role in routine clinical practice.

\section{SUPPORT STATEMENT}

This study was supported by grants from the Programme Hospitalier de Recherche Clinique 2004 (Angers, France), ANTADIR and the Etablissement Public, Chancellerie de Paris (both Paris, France).

\section{STATEMENT OF INTEREST}

Statements of interest for B. Fleury and B. Pételle can be found at www. erj.ersjournals.com $/ \mathrm{misc} /$ statements.dtl

\section{ACKNOWLEDGEMENTS}

We would like to thank: J.M. Gustin (Service de Stomatologie, CHU, Angers, France) and G. Vincent (Service d'ORL, Hôpital Sainte-Antoine, 
Paris, France), dental health professionals, for their advices and technical assistance; the sleep laboratory staff of the Angers University Hospital (Angers) and Hôpital Saint-Antoine (Paris) for their assistance; and A. Bailleul and J.M. Chrétien from the Centre de Recherche Clinique (Angers) for their contribution to data management.

\section{REFERENCES}

1 Young T, Palta M, Dempsey J, et al. The occurrence of sleepdisordered breathing among middle-aged adults. N Engl J Med 1993; 328: 1230-1235.

2 McArdle N, Devereux G, Heidarnejad H, et al. Long-term use of CPAP therapy for sleep apnea/hypopnea syndrome. Am J Respir Crit Care Med 1999; 159: 1108-1114.

3 Engleman HM, Wild MR. Improving CPAP use by patients with the sleep apnoea/hypopnoea syndrome (SAHS). Sleep Med Rev 2003; 7: 81-99.

4 Kushida CA, Morgenthaler TI, Littner MR, et al. Practice parameters for the treatment of snoring and obstructive sleep apnea with oral appliances: an update for 2005. Sleep 2006; 29: 240243.

5 Lim J, Lasserson TJ, Fleetham J, et al. Oral appliances for obstructive sleep apnoea. Cochrane Database Syst Rev 2006; 1: CD004435.

6 Ferguson KA, Cartwright R, Rogers R, et al. Oral appliances for snoring and obstructive sleep apnea: a review. Sleep 2006; 29: 244262.

7 Engleman HM, McDonald JP, Graham D, et al. Randomized crossover trial of two treatments for sleep apnea/hypopnea syndrome: continuous positive airway pressure and mandibular repositioning splint. Am J Respir Crit Care Med 2002; 166: 855-859.

8 Kato J, Isono S, Tanaka A, et al. Dose-dependent effects of mandibular advancement on pharyngeal mechanics and nocturnal oxygenation in patients with sleep-disordered breathing. Chest 2000; 117: 1065-1072.

9 de Almeida FR, Bittencourt LR, de Almeida CI, et al. Effects of mandibular posture on obstructive sleep apnea severity and the temporomandibular joint in patients fitted with an oral appliance. Sleep 2002; 25: 507-513.

10 Gindre L, Gagnadoux F, Meslier N, et al. Mandibular advancement for obstructive sleep apnea: dose effect on apnea, long-term use and tolerance. Respiration. 2008; 76: 386-392.

11 Petelle B, Vincent G, Gagnadoux F, et al. One-night mandibular advancement titration for obstructive sleep apnea syndrome: a pilot study. Am J Respir Crit Care Med 2002; 165: 1150-1153.
12 Pelletier-Fleury N, Meslier N, Gagnadoux F, et al. Economic arguments for the immediate management of moderate-to-severe obstructive sleep apnoea syndrome. Eur Respir J 2004; 23: 53-60.

13 Sleep-related breathing disorders in adults: recommendations for syndrome definition and measurement techniques in clinical research. The Report of an American Academy of Sleep Medicine Task Force. Sleep 1999; 22: 667-689.

14 Johns MW. A new method for measuring daytime sleepiness: the Epworth sleepiness scale. Sleep 1991; 14: 540-545.

15 Mazza S, Pepin JL, Deschaux C, et al. Analysis of error profiles occurring during the OSLER test: a sensitive mean of detecting fluctuations in vigilance in patients with obstructive sleep apnea syndrome. Am J Respir Crit Care Med 2002; 166: 474-478.

16 Verstraeten E, Cluydts R. Executive control of attention in sleep apnea patients: theoretical concepts and methodological considerations. Sleep Med Rev 2004; 8: 257-267.

17 Hoffstein V. Review of oral appliances for treatment of sleepdisordered breathing. Sleep Breath 2007; 11: 1-22.

18 Ferguson KA, Ono T, Lowe AA, et al. A short-term controlled trial of an adjustable oral appliance for the treatment of mild to moderate obstructive sleep apnoea. Thorax 1997; 52: 362-268.

19 Dort LC, Hadjuk E, Remmers JE. Mandibular advancement and obstructive sleep apnoea: a method for determining effective mandibular protrusion. Eur Respir J 2006; 27: 1003-1009.

20 Ferguson KA, Ono T, Lowe AA, et al. A randomized crossover study of an oral appliance vs nasal-continuous positive airway pressure in the treatment of mild-moderate obstructive sleep apnea. Chest 1996; 109: 1269-1275.

21 Tan YK, L'Estrange PR, Luo YM, et al. Mandibular advancement splints and continuous positive airway pressure in patients with obstructive sleep apnoea: a randomized cross-over trial. Eur J Orthod 2002; 24: 239-249.

22 Barnes M, McEvoy RD, Banks S, et al. Efficacy of positive airway pressure and oral appliance in mild to moderate obstructive sleep apnea. Am J Respir Crit Care Med 2004; 170: 656-664.

23 Lam B, Sam K, Mok WY, et al. Randomised study of three nonsurgical treatments in mild to moderate obstructive sleep apnoea. Thorax 2007; 62: 354-359.

24 Clark GT, Blumenfeld I, Yoffe N, et al. A crossover study comparing the efficacy of continuous positive airway pressure with anterior mandibular positioning devices on patients with obstructive sleep apnea. Chest 1996; 109: 1477-1483.

25 Rauscher H, Formanek D, Popp W, et al. Self-reported vs measured compliance with nasal CPAP for obstructive sleep apnea. Chest 1993; 103: 1675-1680. 